\title{
Universiteit
}

Leiden

The Netherlands

\section{Scanning probe microscopes go video rate and beyond}

Rost, M.J.; Crama, L.; Schakel, P.; Tol, E.H. van; Velzen-Williams, G.B.E.M.; Overgauw, C.F.; ... ; Frenken, J.W.M.

\section{Citation}

Rost, M. J., Crama, L., Schakel, P., Tol, E. H. van, Velzen-Williams, G. B. E. M., Overgauw, C. F., ... Frenken, J. W. M. (2005). Scanning probe microscopes go video rate and beyond. Review Of Scientific Instruments, 76(18), 053710. doi:10.1063/1.1915288

Version: $\quad$ Not Applicable (or Unknown)

License: $\quad$ Leiden University Non-exclusive license

Downloaded from: https://hdl.handle.net/1887/61253

Note: To cite this publication please use the final published version (if applicable). 


\section{Scanning probe microscopes go video rate and beyond}

M. J. RostL. Crama, P. Schakel, E. van Tol, G. B. E. M. van Velzen-Williams, and C. F. OvergauwH. ter Horst, H. Dekker, B. Okhuijsen, M. Seynen, and A. VijftigschildP. HanA. J. Katan, K. Schoots, R. Schumm, W. van Loo, T. H. Oosterkamp, and J. W. M. Frenken

Citation: Review of Scientific Instruments 76, 053710 (2005); doi: 10.1063/1.1915288

View online: http://dx.doi.org/10.1063/1.1915288

View Table of Contents: http://aip.scitation.org/toc/rsi/76/5

Published by the American Institute of Physics

\section{Articles you may be interested in}

The ReactorSTM: Atomically resolved scanning tunneling microscopy under high-pressure, high-temperature catalytic reaction conditions

Review of Scientific Instruments 85, 083703 (2014); 10.1063/1.4891811

Tunneling through a controllable vacuum gap

Applied Physics Letters 40, 178 (1998); 10.1063/1.92999

The Topografiner: An Instrument for Measuring Surface Microtopography

Review of Scientific Instruments 43, 999 (2003); 10.1063/1.1685846

The "Reactor STM": A scanning tunneling microscope for investigation of catalytic surfaces at semi-industrial reaction conditions

Review of Scientific Instruments 69, 3879 (1998); 10.1063/1.1149193

Scanning probe image wizard: A toolbox for automated scanning probe microscopy data analysis

Review of Scientific Instruments 84, 113701 (2013); 10.1063/1.4827076

The FAST module: An add-on unit for driving commercial scanning probe microscopes at video rate and beyond Review of Scientific Instruments 82, 053702 (2011); 10.1063/1.3585984

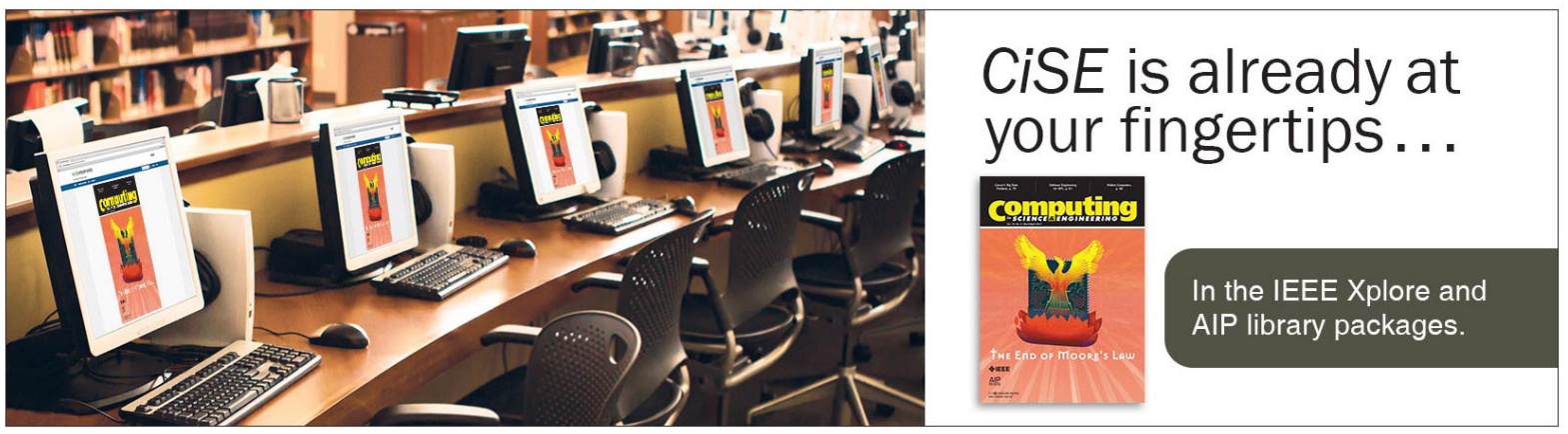




\title{
Scanning probe microscopes go video rate and beyond
}

\author{
M. J. Rost ${ }^{\text {a) }}$ \\ Kamerlingh Onnes Laboratory, Leiden University, P.O. Box 9504, 2300 RA Leiden and Leiden Probe \\ Microscopy BV (LPM), Kamerlingh Onnes Laboratory, Niels Bohrweg 2, 2333 CA Leiden, The Netherlands ${ }^{\text {b) }}$ \\ L. Crama, P. Schakel, E. van Tol, G. B. E. M. van Velzen-Williams, and C. F. Overgauw \\ Kamerlingh Onnes Laboratory, Leiden University, P.O. Box 9504, 2300 RA Leiden, The Netherlands \\ H. ter Horst, H. Dekker, B. Okhuijsen, M. Seynen, and A. Vijftigschild \\ FOM-Institute for Atomic and Molecular Physics, Kruislaan 407, 1098 SJ Amsterdam, The Netherlands \\ P. Han \\ The Pennsylvania State University, University Park, Pennsylvania 16802-6300
}

A. J. Katan, K. Schoots, R. Schumm, W. van Loo,

T. H. Oosterkamp, and J. W. M. Frenken

Kamerlingh Onnes Laboratory, Leiden University, P.O. Box 9504, 2300 RA Leiden, The Netherlands

(Received 18 February 2005; accepted 8 March 2005; published online 26 April 2005)

In this article we introduce a, video-rate, control system that can be used with any type of scanning probe microscope, and that allows frame rates up to 200 images/s. These electronics are capable of measuring in a fast, completely analog mode as well as in the more conventional digital mode. The latter allows measurements at low speeds and options, such as, e.g., atom manipulation, currentvoltage spectroscopy, or force-distance curves. For scanning tunneling microscope (STM) application we implemented a hybrid mode between the well-known constant-height and constant-current modes. This hybrid mode not only increases the maximum speed at which the surface can be imaged, but also improves the resolution at lower speeds. Acceptable image quality at high speeds could only be obtained by pushing the performance of each individual part of the electronics to its limit: we developed a preamplifier with a bandwidth of $600 \mathrm{kHz}$, a feedback electronics with a bandwidth of $1 \mathrm{MHz}$, a home-built bus structure for the fast data transfer, fast analog to digital converters, and low-noise drivers. Future improvements and extensions to the control electronics can be realized easily and quickly, because of its open architecture with its modular plug-in units. In the second part of this article we show our high-speed results. The ultrahigh vacuum application of these control electronics on our (UHV)-STM enabled imaging speeds up to $0.3 \mathrm{~mm} / \mathrm{s}$, while still obtaining atomic step resolution. At high frame rates, the images suffered from noticeable distortions, which we have been able to analyze by virtue of the unique access to the error $(d Z)$ signal. The distortions have all been associated with mechanical resonances in the scan head of the UHV-STM. In order to reduce such resonance effects, we have designed and built a scan head with high resonance frequencies $(\geqslant 64 \mathrm{kHz})$, especially for the purpose of testing the fast electronics. Using this scanner we have reached video-rate imaging speeds up to 200 frames/s (5 ms/image), while still obtaining atomically resolved structures. () 2005 American Institute of Physics. [DOI: 10.1063/1.1915288]

\section{INTRODUCTION}

Inspired by the pioneering work by Young et al. ${ }^{1}$ on scanning probe microscopy (SPM), Binnig and Rohrer invented the scanning tunneling microscope (STM) in 1982.,3 This technique has developed to become one of the most important instruments for real-space surface observations on small length scales. The broad capabilities of SPM technology are clearly reflected in the number of applications on various samples and at different conditions. While the samples range from single- and polycrystalline metals to in-

\footnotetext{
a) Author to whom correspondence should be addressed; electronic mail: rost@physics.leidenuniv.nl

${ }^{b)}$ www.leidenprobemicroscopy.com
}

sulators and a wide variety of biological materials, such as membranes, DNA, and other molecules, the conditions range from extreme temperatures, ${ }^{4,5}$ real variable temperature, ${ }^{6}$ vacuum or ambient to high pressures, ${ }^{7,8}$ or even measurements in electrolytic solution. In addition, access to different physical properties has been acquired by the development of various alternative sensing methods [e.g., atomic force microscopy (AFM), magnetic force microscopy, spin polarized STM, scanning near field optical microscopy,...]. Also industry has recognized the power of SPM technology: industrial research projects are financed to investigate possibilities for, e.g., data storage, ${ }^{9}$ scanning lithography,${ }^{10}$ catalysis, ${ }^{11}$ and quality control. ${ }^{12}$

Although the strengths of SPMs are obvious, there are 
still clear drawbacks on many aspects, most notably on the image recording time (speed) and on the user friendliness with respect to the control of such an instrument. The slow speed of SPMs limits (on the one hand) researchers in their studies of fast dynamical processes, especially on samples that prohibit a temperature decrease (e.g., many biological samples), and limits (on the other hand) industry in its application of this technology within their fabrication lines for quality control. In addition, the complex and refined control of the present-day SPM instruments requires experienced operators so that high-quality data are obtained without damage to either the probe or the sample.

When trying to speed up SPM measurements, ${ }^{13-19}$ one faces two main challenging problems. The first is the necessity of a very stable mechanical loop between the probe and the sample, ${ }^{20}$ which requires a scan head with high resonance frequencies. In all SPMs, in which it is possible to excite vibrations (resonances) either by the scanning motion or by external mechanical or acoustical noise, high-speed measurements are strongly impaired or even completely impossible due to resolution problems. In the worst case, this can even lead to a tip crash (collision between the tip and the sample) that destroys the probe and/or the sample.

The second challenge concerns the control system. ${ }^{21}$ All components of the feedback electronics ${ }^{22}$ have to be sufficiently high in bandwidth (including appropriately high slew rates) as well as sufficiently low in noise. ${ }^{23}$ These conditions are conflicting, as the noise of electronic components usually increases with the bandwidth $f_{\mathrm{bw}}$ proportional to $\sqrt{f_{\mathrm{bw}}}$. In addition, one also needs a low-noise scan generator, a fast data acquisition system, and fast display of the measured data.

In this article we describe our approach, which includes improvements on all the above-mentioned aspects of SPM technology. The first part describes the development of a SPM control system that allows high-speed scanning and data acquisition. In the second part we use this control system for initial high tip-speed observations with our existing variable temperature ultrahigh vacuum (UHV)-STM. ${ }^{24} \mathrm{Al}-$ though we reach tip speeds of $0.3 \mathrm{~mm} / \mathrm{s}$, while still obtaining atomic step resolution, we clearly face the limits of the scan head of this STM: while increasing the imaging speed, we observe increasingly strong image distortions. We analyze these distortions and relate them to the mechanical behavior of the scanner. On the basis of these insights, we have constructed a high-resonance-frequency scanner that we present in the third part of this article. With this scanner we reach measuring speeds up to $5 \mathrm{~ms}$ per image (200 images/s with $256 \times 32$ pixels per image) and line rates up to $10.2 \mathrm{kHz}$ ( 80 images $/ \mathrm{s}$ with $128 \times 128$ pixels), while still obtaining atomic resolution on highly oriented pyrolitic graphite (HOPG).

\section{HIGH-SPEED SPM CONTROL SYSTEM}

Considering high-speed SPM measurements we have formulated the following design principles and requirements:

(1) The control system must be capable of measuring full-size SPM images $(256 \times 256$ pixels $)$ at video rate (25 images/s), while providing full, on-line control over the scan parameters and the feedback settings.

(2) The scan generation must be "as analog as possible" (in contrast to the typical digital scan generators), in order to avoid the excitation of mechanical resonances resulting from the discretization noise in the scan signals.

(3) A digital scan generation option must also be implemented in order to facilitate ultraslow scans, atom manipulation, and current-voltage measurements.

(4) The feedback electronics, including all components, must have a minimum bandwidth of $600 \mathrm{kHz}$, which is necessary for atomically resolved imaging at video rate ( 25 frames/s) for frame sizes of $256 \times 256$ pixels.

(5) The feedback electronics must be capable of measuring the error signal in addition to the control signal.

(6) The control system must be sufficiently versatile to provide easy connection with any type of SPM.

(7) All SPM-control and measurement signals must be directly accessible to guarantee openness to a wide variety of alternative configurations and extensions, e.g., signal modulation,....

(8) The control system must consist of standard plug-in units $^{25}$ to allow easy upgrades and repairs, without the need of to replace larger parts of the electronics.

(9) A modular architecture enables setup of specific solutions via the interchange of only a few plug-ins.

(10) The operating system should be wINDOWS/PC based, since this platform provides excellent continuity, support, and compatibility. However, in order to make the electronics independent of the specific choice of computer and operating system, a home-developed control/data bus is necessary.

(11) Our minimum demand is to measure with an imaging rate of $25 \mathrm{~Hz}(256 \times 256$ pixels per image). For every line from left to right (trace) there must be a line from right to left (retrace). In addition, we record two signals: the tip height control signal and the residual error signal. Together, this leads to an additional factor of 4 , so that our system requires a data-bus rate of $13 \mathrm{Msamples} / \mathrm{s}$, where each sample represents a 16 bit value.

Figure 1 shows a schematic of the control system. It consists of four 19 in. crates: (i) the feedback, (ii) the scan generator, (iii) the analog to digital converters (ADCs), and (iv) the drivers. Each 19 in. crate is built from standard components and consist of standard plug-ins. ${ }^{25}$ The use of these plug-ins allows the separation of each basic function, and provides the versatility to use the electronics for, in principle, any kind of SPM. Moreover, it also guarantees that the electronics can be easily updated or repaired. All signal connections between the individual plug-ins are balanced, in order to obtain a high degree of common-mode rejection. Since the user can (has to) make all these connections via the front panels, the user automatically has direct access to all signals. In the following section we will describe the four crates.

\section{A. Feedback electronics}

The feedback crate (see Fig. 1) consists of four plug-ins, which we call an input converter (in our case a tunneling 


\begin{tabular}{|c|c|c|c|}
\hline Drivers & ADC-rack & Scan-rack & Feedback \\
\hline \multirow{2}{*}{ X-Driver } & ADC Bus & Scan Bus & Monitor \\
\hline & Z-ADC & X-Generator & STM-Input \\
\hline \multirow{2}{*}{ Y-Driver } & $\mathrm{dZ}-\mathrm{ADC}$ & Sine-Gen. & P/l/Filter \\
\hline & I-ADC & Y-Generator & Outputs \\
\hline \multirow{2}{*}{ Z-Driver } & $\cdots$ & X'-Mixer & $\cdots$ \\
\hline & $\ldots$ & Y'-Mixer & $\ldots$ \\
\hline \multirow{2}{*}{ P-Driver } & $\cdots$ & Z-Mixer & $\cdots$ \\
\hline & $\cdots$ & Waveform & $\ldots$ \\
\hline \multirow{2}{*}{$\cdots$} & $\cdots$ & U-sample & $\cdots$ \\
\hline & $\ldots$ & Readout & $\cdots$ \\
\hline \multirow[b]{2}{*}{$\cdots$} & $\cdots$ & Output & $\cdots$ \\
\hline & $\ldots$ & Input & $\ldots$ \\
\hline
\end{tabular}

FIG. 1. The control system consists of four 19 in. crates: the feedback, the scan generator, the ADCs, and the drivers, (from top to bottom). A current preamplifier is indicated for the application of the control system to STM.

current input converter), a $P / I /$ filter module, which is combined with an output that delivers the measurement signals, and a monitor. The monitor provides access to the input signal, e.g., the tunneling current in STM can be monitored directly on an oscilloscope. In addition, it is also possible to "listen" to the signal by using a headphone connector on the monitor. The STM input plug-in converts the tunneling current signal that comes from a preamplifier (in the form of a voltage) into a standardized signal; it consists of an absolute value amplifier (ABS) and a logarithmic converter. In order to use the control system for other SPMs, this input converter plug-in can be exchanged: for example, we have developed an input converter for intermittent-contact mode AFM. The standardized signal of the input converter goes through the $P / I /$ filter module, where a reference value is subtracted (corresponding to the setpoint of, e.g., the tunneling current), the proportional and integral gains are set, and the filter bandwidth is chosen. The output plug-in delivers all necessary output signals, both for the drivers and for the ADCs.

Shown at the top of Fig. 1 is one of the most important devices of STM feedback electronics, namely the current preamplifier. $^{26}$ We will first present our preamplifier improvements, before discussing the rest of the feedback circuit in more detail.

\section{Preamplifier}

The noise of an opamp that is used as a current-tovoltage converter contains three distinct sources: current noise, voltage noise, and Johnson (thermal) noise of the feedback resistor. As the frequency dependence varies with the type of noise, one has to either specify each noise source separately, or characterize the frequency-integrated noise as a function of bandwidth. Figure 2 shows the equivalent integrated input current noise of our home-built 50 and $600 \mathrm{kHz}$ preamplifiers. The measurements were performed with a

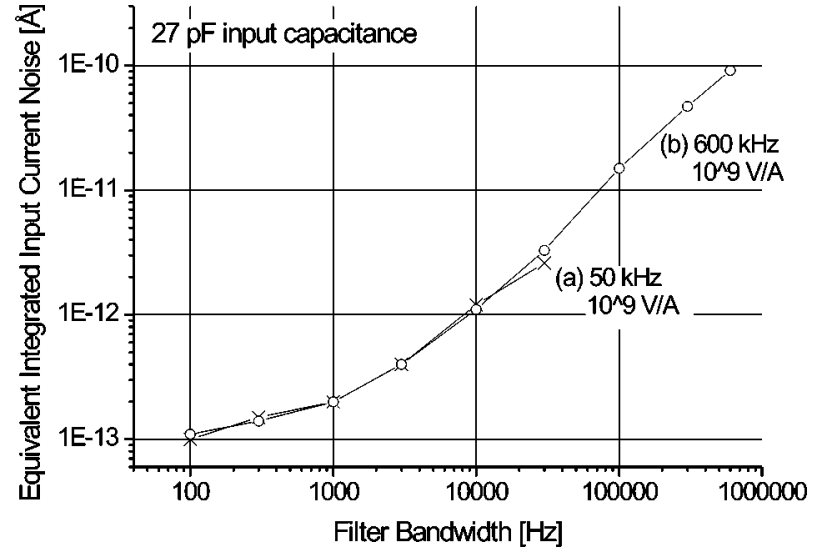

FIG. 2. Equivalent integrated input current noise of our: (a) $50 \mathrm{kHz}$ and (b) $600 \mathrm{kHz}$ home-built preamplifiers, both with a capacitive input load of $27 \mathrm{pF}$.

fourth-order low-pass filter. A comparison of our preamplifiers with several other commercially available ones ${ }^{27}$ revealed that the performance of our preamplifiers was significantly superior. For example, the commercial preamplifiers with a bandwidth comparable to ours showed a noise level that is between four and ten times higher than ours. Note that a fair comparison between preamplifiers that operate with different resistor gains requires the conversion of the measured output noise back to the equivalent input current noise. In general, it is impossible to reach a fA noise level with a large bandwidth: these conditions are conflicting. Since the noise of our $600 \mathrm{kHz}$ preamplifier at low frequencies is as low as the noise of the $50 \mathrm{kHz}$ preamplifier at those frequencies, it is possible to use only the $600 \mathrm{kHz}$ preamplifier with a switchable low-pass filter. ${ }^{28}$ As an example, we obtain a noise level of $\sim 1 \mathrm{pA}$, when using a filter frequency of $10 \mathrm{kHz}$.

Both preamplifiers have been designed on the basis of field effect transistor (FET) inputs, where great care has been taken to reach optimal component matching. In addition, both preamplifiers are equipped with a compensation circuit. It also turned out that the power supply noise is of great importance for the noise performance. The combination of careful component choices, theoretical calculations, electronic simulations, and comparison with experimental performance has allowed us to successfully construct the low-noise $600 \mathrm{kHz}$ version.

\section{Feedback}

In comparison with conventional control systems, we have realized a feedback concept that allows us to measure in a hybrid mode between the familiar constant height and constant current modes: the idea is not only to make use of the height signal, but also to measure simultaneously the deviation of the tip with respect to the reference (requested) tip height. ${ }^{29}$ Measuring an error signal is common in AFM technology, but the transformation of this signal to additional height information is often not straightforward, because of its highly nonlinear dependence.

As we show here, in STM technology, it is possible to accurately measure the height of the tip simultaneously with 


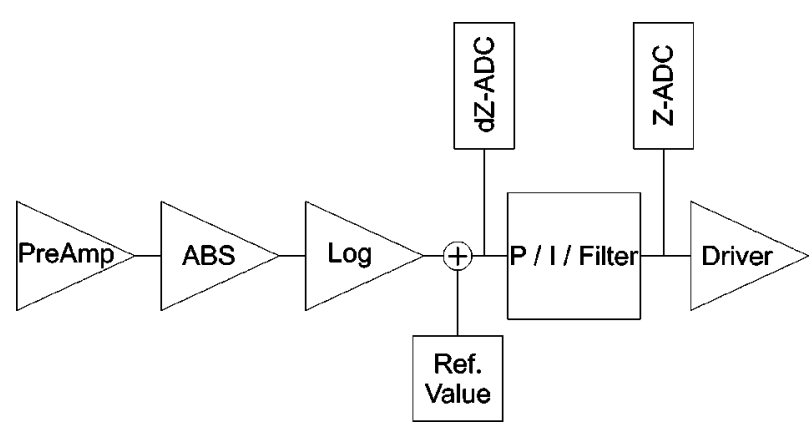

FIG. 3. Schematic of our feedback circuit including the hybrid mode option. After the amplification of the tunneling current by the preamplifier, which is located close to the scan head, the signal runs through an absolute value amplifier (ABS), and a logarithmic amplifier (Log), before it is compared with the reference value. From there, the error signal passes through a unit that consists of a proportional gain, an integral gain, and a filter, before it is amplified by the high-voltage driver and supplied to the $Z$-electrode of the piezo element.

its error with respect to its ideal height (setpoint or reference height). This makes it possible to calculate back the true surface contour, ${ }^{30}$ even at high tip speeds, when the feedback settings are such that the tip is not capable of following the surface accurately.

Which signals provide the most accurate information for the hybrid mode? Figure 3 shows a schematic of the complete feedback circuit. The most straightforward piece of height information of the tip is given by the output voltage of the feedback electronics (see the position of Z-ADC in Fig. 3 ), since this signal is further only amplified by the highvoltage driver and then directly connected to the piezo element. As will be shown later, this signal does not necessarily represent the actual tip height, as the piezo response can lag behind the driving voltage.

Since the feedback gain is finite for all frequencies (except for dc), the error signal always differs from zero during the scanning motion of the tip. This also means that the tip trajectory deviates for all imaging speeds (except for zero) from the perfect height contour that would correspond to the truly constant current condition. The magnitude of this deviation increases with higher imaging speeds, since the closed loop gain decreases for higher frequencies. ${ }^{31}$ It is possible to accurately measure this deviation in the tunneling current. Since the tunneling current varies exponentially with the distance between the tip and the sample, the most appropriate way to detect the error is to measure the signal after a logarithmic conversion and a comparison with a reference point (see dZ-ADC in Fig. 3). This error signal $d Z$ depends linearly on the height error.

This hybrid mode allows us to measure the surface topography in a different way than usual. On a macroscopic scale we can roughly follow the surface structure in order to stay in tunneling range, while we obtain the detailed (atomic) information via the variation in the error signal $(d Z)$. Using our broadband $600 \mathrm{kHz}$ preamplifier without a filter, $d Z$ is also measured with a bandwidth of $600 \mathrm{kHz}$, while the bandwidth of the closed loop can be smoothly tuned between 1 and $100 \mathrm{kHz}$ (see $P / I /$ filter in Fig. 3). Note that for a meaningful recording of the error signal, the noise at the full

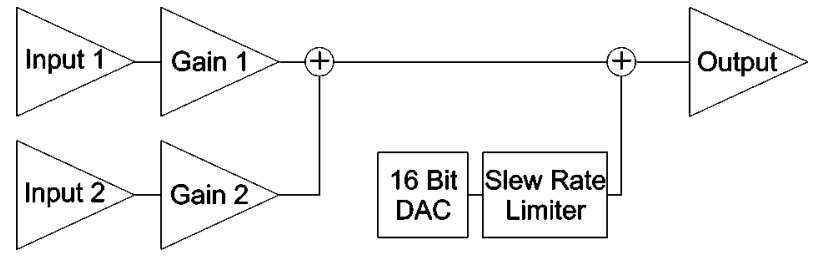

FIG. 4. Mixer concept: two input channels, e.g., $X$ and $Y$, and the output from a 16-bit DAC are added up to provide a combined signal.

$600 \mathrm{kHz}$ bandwidth has to be minimal, which has become possible by virtue of a very careful selection of all electrical components in the feedback circuit.

\section{B. Scan generator}

In contrast to most SPM control systems, we have developed a completely analog scan generator ${ }^{32}$ that can be controlled digitally. In addition, the scan generator is also capable of scanning in a purely digital mode. By using the analog scan generation we avoid all common digital problems, like glitches or high-frequency crosstalk. Moreover, the advantages of analog and digital electronics have been combined: user comfort is guaranteed by the computercontrolled access. A second advantage of the analog scan generation is that the number of pixels is independent of the scan size (at least along the line direction). Large-area scans with atomic resolution are possible in this way. One can, e.g., measure a $1 \mu \mathrm{m}$ large area with 10000 pixels/line so that one still achieves a resolution of $1 \AA$ / pixel.

The second crate in Fig. 1 contains the individual plugins of the scan generator. The scan bus connects this rack via optical fibers to the computer. The $X$ generator is a completely analog scan generator of linear ramps (triangular wave form), with possible line frequencies between $0.1 \mathrm{~Hz}$ and $10 \mathrm{kHz}$. This generator can round off the triangle tops by smoothly connected parabola (see below): the degree of rounding is adjustable via the software. The $Y$ generator works on a digital basis with integrators that smoothe the voltage steps linearly. The integration time, which is set by the computer, is automatically calculated by the software in order to match the user's choice of tip speed. The $X$ and the $Y$ generator signals are combined via mixer modules: the $X^{\prime}$ mixer, the $Y^{\prime}$ mixer, and the $Z$ mixer. Figure 4 shows a schematic of one mixer. Each mixer combines two input channels, each of which is amplified (Gain1 and Gain2 in Fig. 4) separately, before they are added up. In addition, a 16-bit digital to analog converter (DAC) signal that is limited in its slew rate is added to the result. Both the amplification factors (Gain1 and Gain2) and the slew rate limiter are adjustable via the computer.

For regular scanning operation the $X$ - and the $Y$-generator outputs serve as the two inputs signals for both the $X^{\prime}$ mixer and the $Y^{\prime}$ mixer. These two mixers are used to perform scan rotations, using appropriate combinations of the four adjustable input gains. They are also used to perform panning via the 16-bit DAC. Both mixers offer two identical outputs. One output is typically connected to the respective driver ( $X$ driver, $Y$ driver), whereas the other is connected to one of the two inputs of the $Z$ mixer (see below). 
The $Z$ mixer fulfills a special task, since the height of the tip is not only controlled by the feedback electronics, but also by this mixer. By using the 16-bit DAC offset we can compensate for any offset value of the feedback electronics. In addition, by using the scan signals as inputs of the $Z$ mixer, we can also compensate for the average tilt of the sample. The working point of the feedback electronics is, therefore, around $0 \mathrm{~V}$. This has a beneficial effect both on the bandwidth of our loop, which increases, and on the signal to noise ratio of the height signal $(Z)$, because the near-zero value of the error signal allows us to use a high, additional amplification in front of the Z-ADC (up to a factor 64).

As will be explained below, stable scanning with very high line frequencies is only possible by using a true sine wave form instead of a triangle. Therefore we also added a sine-wave form generator, which can be exchanged with the $X$ generator.

The programmable wave form generator is used to deliver ramps for piezo motors. ${ }^{33}$ Potentiometer positions, e.g., set point tunneling current, proportional gain, or integrator gain, are digitized via the readout card, which enables the control system to store them together with the measured data. An output plug-in, which can be used to feed out signals, allows conversion from differential connections that are used in the electronics to single ended BNC-connectors. By analogy, the input plug-in provides the possibility to feed external signals into the electronics.

\section{ADC crate}

The ADC crate consists of an ADC-bus module for the data transfer and 16-bit ADCs. Depending on the specific application this crate can be fitted with up to eight ADCs, which can all be read out simultaneously. Each of the ADCs is equipped with an internal gain that is controlled via the software to a value of $1 \times, 2 \times, 4 \times, 8 \times, 16 \times, 32 \times$, or $64 \times$. This provides us with a $Z$ accuracy of $0.002 \AA$, when using a $13 \mathrm{~mm}$ long EBL2 piezo tube.

\section{Driver crate}

The driver crate can be fitted with up to six high-voltage drivers. Typically, we use four drivers: $X, Y, Z$, and one for a piezo motor. All drivers provide two fully equivalent input channels that are added before amplification. This possibility is in particular important for the $Z$-driver, as we use it for the $Z$-offset correction, which improves the signal to noise ratio and the bandwidth of the feedback electronics (see above). With an adjustable amplification between $15 \times$ and $19 \times$, and inputs between $\pm 10 \mathrm{~V}$, the drivers are capable of delivering a balanced, maximum output voltage of $\pm 190 \mathrm{~V}$. Figure 5 shows the integrated rms voltage noise as a function of filter cutoff frequency for a capacitive load of $1.5 \mathrm{nF}$. The smallsignal bandwidth of the drivers is $400 \mathrm{kHz}$, and the fullpower bandwidth decreases to only $150 \mathrm{kHz}$. This is due to the very high slew rate, which amounts to $75 \mathrm{~V} / \mu$ s for capacitive loads up to $1 \mathrm{nF}$ and to $50 \mathrm{~V} / \mu$ s for a load of $4.7 \mathrm{nF}$. The buzz $(50 \mathrm{~Hz})$ is only $3 \mu \mathrm{V} \mathrm{rms}$. These drivers

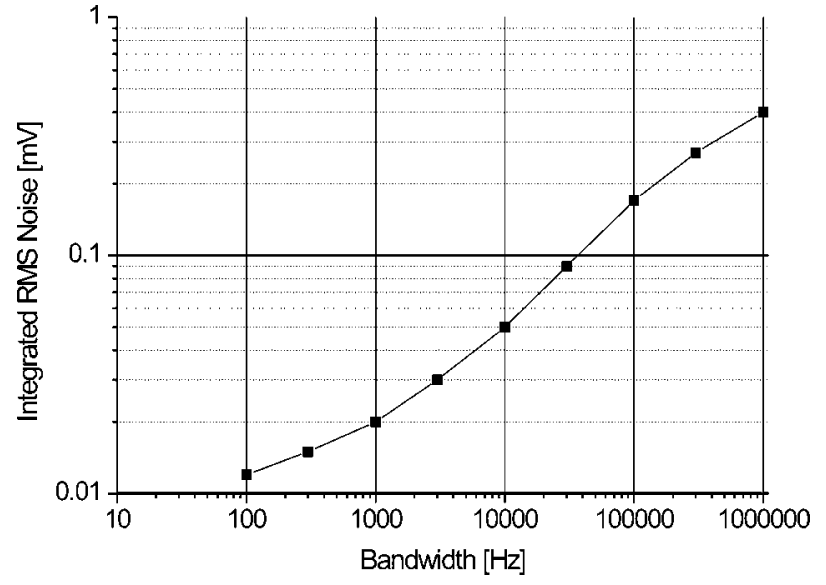

FIG. 5. Integrated rms voltage noise of the drivers with a capacitive load of $1.5 \mathrm{nF}$.

are built on the basis of standard technology, and their excellent performance is the result of a careful design and a careful choice and matching of the components.

\section{RESULTS}

This section describes the results of our first high-speed measurements. We have used the electronics, described in the previous section, to control several STMs and AFMs. In each case we observed an improvement in imaging speed and resolution, when compared to commercial or oldergeneration home-built electronics. ${ }^{15}$

At sufficiently high tip speeds we observe imaging problems for all SPMs. The access to the error signal $(d Z)$ allows us to quantify these problems. We have compared the outcome of this with the results of a mechanical study of our scanner. From this analysis we have obtained information on the real tip movement that deviates from the ideal behavior, corresponding to the applied controlling voltages.

\section{A. Standard UHV scan head \\ 1. Improved resolution}

We have performed the first test of the control system by connecting it to our UHV variable-temperature STM. ${ }^{24}$ Figure 6(a) shows the regular height signal on a $\mathrm{Cu}(001)$ surface: monoatomic steps are visible. Simultaneously, we have measured the deviation or error signal $(d Z)$, which is displayed in Fig. 6(b). If the tip would have followed the ideal constant-current height contour, Fig. 6(b) would contain no information, since the error would be zero everywhere. Clearly, this is not the case, which reflects that there are restrictions on the gain of the feedback loop (see above) as well as on the mechanical behavior of the scanner. Since our error signal is proportional to the actual height error, we can easily calculate the real surface contour by multiplying the values in Fig. 6(b) with the appropriate proportionality constant and adding the result to the heights of Fig. 6(a). The outcome of this operation is displayed in Fig. 6(c). Notice the enhanced contrast both on the step edges and on the impurities in the terraces. In addition, a mechanical resonance of the scanner is visible in the error signal [top half of 

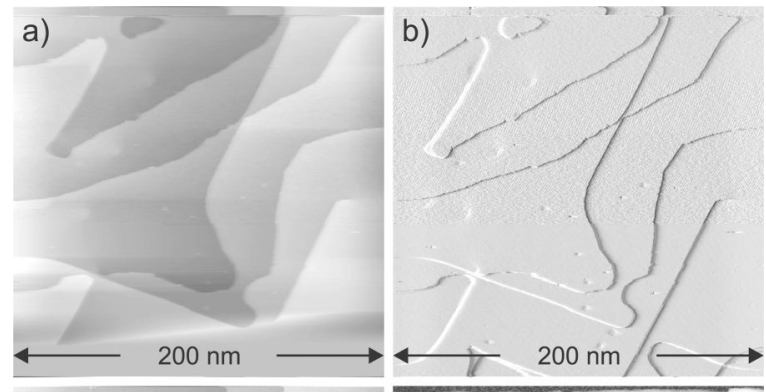

c)

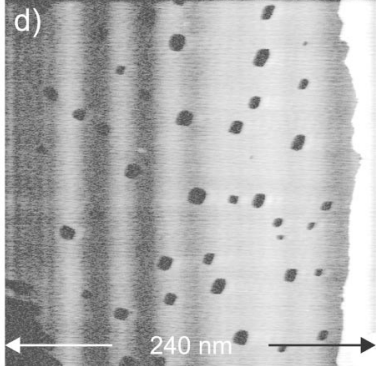

FIG. 6. STM images $(512 \times 512$ pixels $)$ on the $\mathrm{Cu}(001)$ surface: (a) standard height image, measured with $3 \mathrm{~Hz}$ line rate; (b) simultaneously measured error $(d Z)$ signal; (c) real surface contour computed from panels (a) and (b); and (d) monoatomic vacancy islands observed at a tip speed of $0.3 \mathrm{~mm} / \mathrm{s}$ (586 Hz line rate). All images were acquired at a sample voltage of $-0.7 \mathrm{~V}$ and tunneling currents below $0.1 \mathrm{nA}$

Fig. 6(b)]. This resonance appeared during tuning of the feedback gain settings, to the maximum stable settings, i.e., just below resonance, in order to maximize the resolution. We find that the resonance is first visible in the error signal $(d Z)$, before it also appears in the height image at higher gain settings.

\section{High tip speeds: $0.3 \mathrm{~mm} / \mathrm{s}$}

Figure 6(d) shows a STM image of a $\mathrm{Cu}(001)$ surface that we had prepared with several, monoatomically deep, vacancy islands. This image was taken from a movie that can be downloaded from Ref. 34. The tip speed during this measurement was $0.3 \mathrm{~mm} / \mathrm{s}$ - a truly macroscopic velocitywhile we still obtained atomic step resolution. In the movie you can also see how real-time panning is performed without any loss in imaging sped and image quality. We will return to the characteristic of the distortions at the left side of the image (movie) in Secs. III C and III D.

\section{Mechanical analysis}

In order to interpret the above mentioned image distortions, we performed a mechanical analysis of our scanner. It was reported earlier ${ }^{35}$ that it is possible to analyze the mechanical behavior of piezo tubes, other scan devices, or even complete scan heads, by applying a sinusoidal excitation to one or a combination of several electrodes of the piezo element and simultaneously measuring the response at (an)other appropriate electrode(s) as a function of the excitation frequency.

Here, we introduce a method that allows us to use the same electrode(s) simultaneously for both the excitation of mechanical vibrations and the measurement of the response. To this end we make use of the resonance/antiresonance behavior that is introduced in the capacity of a piezoelectrical

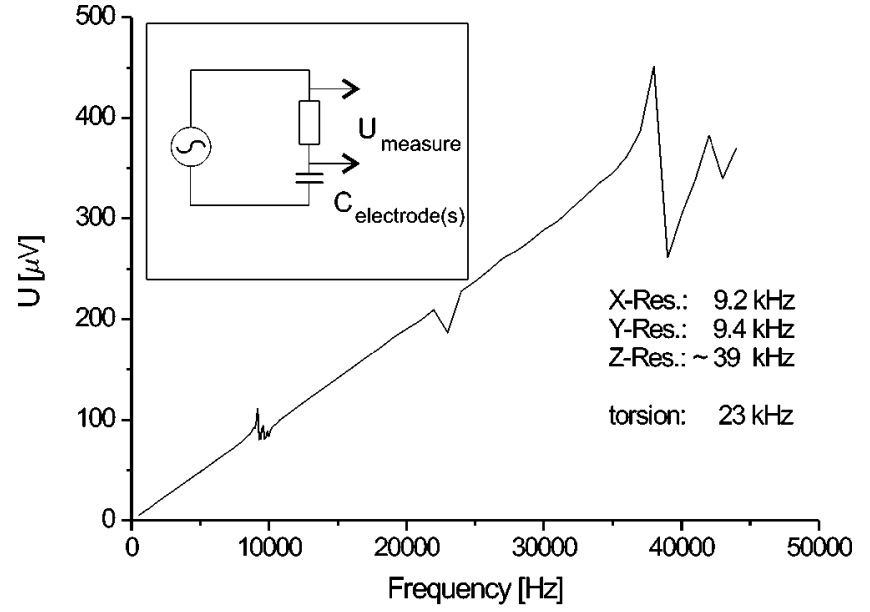

FIG. 7. Mechanical analysis of our UHV scanner.

element, when this passes through one of its mechanical resonance frequencies. The inset in Fig. 7 shows a schematic. The response amplitude was measured with a lock-in amplifier.

Applying this technique to different electrodes and their combinations, one clearly can distinguish between different eigenmodes. As an example, Fig. 7 shows a measurement, where we use only one $X$ electrode of the piezo tube of our UHV scanner. We find that the first bending mode in the $X$ direction appears at $9.2 \mathrm{kHz}$, whereas the corresponding mode in the $Y$ direction lies at $9.4 \mathrm{kHz}$, as found in a similar measurement with one $Y$ electrode. The reason for this broken rotational symmetry lies in the lack of precise symmetry of our scan head which, e.g., rests on three legs ${ }^{24}$ and has the electrical connection for the tunneling current mounted on one side. The first longitudinal mode ( $Z$ direction) is at $\sim 38.5 \mathrm{kHz}$, and the peak at $23 \mathrm{kHz}$ corresponds to the first torsional mode of the piezo tube.

We verified that these frequencies correspond very closely with the results of finite element calculations, ${ }^{36}$ where we included the details of our specific scanner geometry and the relevant material parameters of our tip holder.

\section{Distortions at higher speeds}

Let us focus here on the image distortions that we observe at high scan speeds or, more importantly, at high line frequencies. While we increase the scanning speed, we clearly pass through frequency regimes of very high distortion, where it is difficult to extract meaningful information from the images. Between these frequency regimes of excessive distortion, we obtain almost perfect images of the surface as we would obtain at low scan speeds. In the following we will show that these frequency regimes of high distortion correspond to the excitation of mechanical eigenmodes of the scanner. In some cases the excitation leads to a tip and/or surface damaging tip crash.

Figure 8 shows six images that are taken from a movie (which can be downloaded from Ref. 34), in which we continuously increased the line frequency from $343 \mathrm{~Hz}$ to $1.5 \mathrm{kHz}$. All images show four monoatomic deep vacancy islands on the $\mathrm{Cu}(001)$ surface. Notice both the vertical distortion bands in the images and the shape of the 

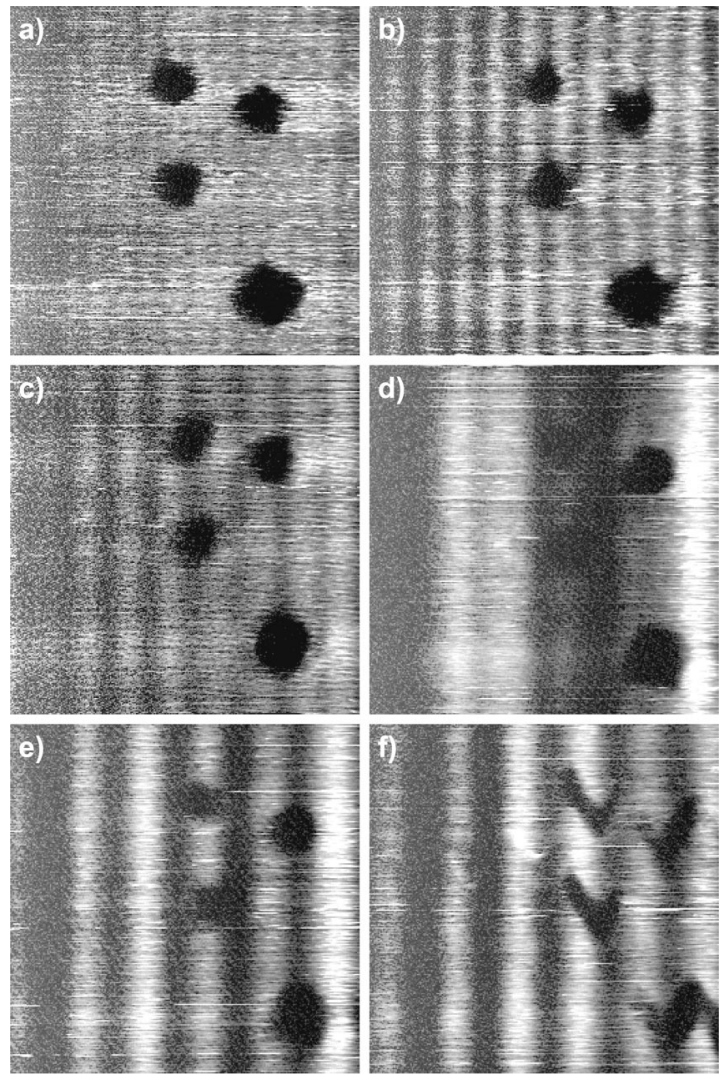

FIG. 8. STM images of four vacancy islands on a $\mathrm{Cu}(001)$ surface at different frame/line rates (see Table I): size $50 \mathrm{~nm}\left(256 \times 256\right.$ pixels, $U_{s}$ $=-0.7 \mathrm{~V}$, and $\mathrm{I} \leqslant 0.1 \mathrm{nA})$.

vacancy islands, which deviates from their undistorted, rounded, square-like form. The distortions can even be so large that the vacancies are "smiling" at you [see Fig. 8(f)]. In order to explain the observed image distortions, we note that it must be possible to excite the eigenmodes of the scan head by the fast scanning motion of the tip, in particular by the turning points between the lines of the trace (left-right) and the retrace (right-left) images. These points correspond to the sharp peaks of a triangular wave form, and it is obvious that they introduce higher Fourier components to the scanner than just the line frequency, which is the fundamental frequency of the triangular wave form. In addition, excitation of an eigenmode, might also be possible via the feedback electronics, when a regular surface pattern is scanned with a tip speed that corresponds to the frequency of that eigenmode of the scan head. Although the feedback controls only the height $(Z)$ motion of the piezo, excitation of differently polarized eigenmodes (bending, rotation,...) is always possible, due to coupling terms in the mechanical response of the piezo element.

It is important to realize that in the present example of a STM scanner in UHV, all eigenmodes have a very high quality $(Q)$ factor, on the order of 100 . As a consequence, the frequency widths of the resonance peaks are small. This means that only frequencies, which are in the near vicinity of a resonance, can excite the eigenmode. On the other hand, once an eigenmode is excited, it takes a long time before it is damped out.

The above described picture immediately explains the
TABLE I. Measured frequencies of strong image distortion.

\begin{tabular}{lccc}
\hline \hline Figure & Frame rate $(\mathrm{Hz})$ & Line rate $(\mathrm{Hz})$ & Distortion $(\mathrm{kHz})$ \\
\hline $8(\mathrm{a})$ & 1.3351 & 341.8 & 9.3 \\
$8(\mathrm{~b})$ & 1.7166 & 439.4 & 9.2 \\
$8(\mathrm{c})$ & 2.2888 & 585.9 & 9.6 \\
$8(\mathrm{~d})$ & 3.0517 & 781.3 & 3.0 \\
$8(\mathrm{~d})$ & 3.0517 & 781.3 & 9.4 \\
$8(\mathrm{e})$ & 3.0899 & 791.0 & 9.0 \\
$8(\mathrm{f})$ & 3.3150 & 848.6 & 9.5 \\
$6(\mathrm{~d})$ & 1.1444 & 585.9 & 38.7 \\
$6(\mathrm{~d})$ & 1.1444 & 585.9 & 8.8 \\
\hline \hline
\end{tabular}

observed behavior, where we pass several regimes of very high distortion during a sweep of the frequency: the distortions appear only if the line frequency (or one of its higher harmonics) is equal to or close to a mechanical eigenmode. We have expressed the observed distortions in terms of frequencies (shown in Table I), to find that these frequencies all indeed perfectly match the mechanical eigenmodes of the scanner. Note that both the bending mode and the longitudinal $(Z)$ mode are excited in Fig. 6(d) (visible on the left side of the image). Figure 8(d) also shows two frequencies: 9.4 and $3.0 \mathrm{kHz}$. The first clearly belongs to the bending mode of the piezo tube, whereas the latter is attributed to a mechanical eigenmode of the scan head.

We now come back to the "smilies." It is obvious that we can excite the bending mode in the direction of the fast scanning motion ( $X$ direction in our case). But is also possible for the fast $X$ motion to excite the perpendicular bending mode ( $Y$ direction), which becomes very clear in the present example where the two modes differ in frequency; the maximum $Y$ distortion is to be expected when the fast scanning motion matches this $Y$ resonance. The "smilies" are obtained, when the scanning frequency (or one of its higher harmonics) lies inbetween the $X$ and the $Y$ mode, i.e., between 9.2 and $9.4 \mathrm{kHz}$, so that both modes are excited. In this case the tip does not follow a (modulated) straight-line trajectory. Instead, it follows a path that is modulated with sine waves in both the $X$ direction and the $Y$ direction.

There are two approaches to reduce the mechanical resonance problems in fast SPMs. The first improvement can be booked by introducing smart scanning signals that avoid high frequency components. The other approach is to introduce new designs of the scan head, with much higher resonance frequencies. The following subsections describe both approaches.

\section{Smart scan signals}

The gray line in Fig. 9 shows the purely triangular analog scan voltage, applied in the fast scanning direction. Obviously, this signal contains Fourier components at much higher frequency than the fundamental frequency of the triangular wave form itself. This can be seen in the Fourier analysis, where even the ninth harmonics is visible. Precisely, these higher harmonics are the real problem, since they excite the eigenmodes, as described above. In order to reduce the amplitude of these harmonics, we electronically implemented the possibility to round the tops of the analog 


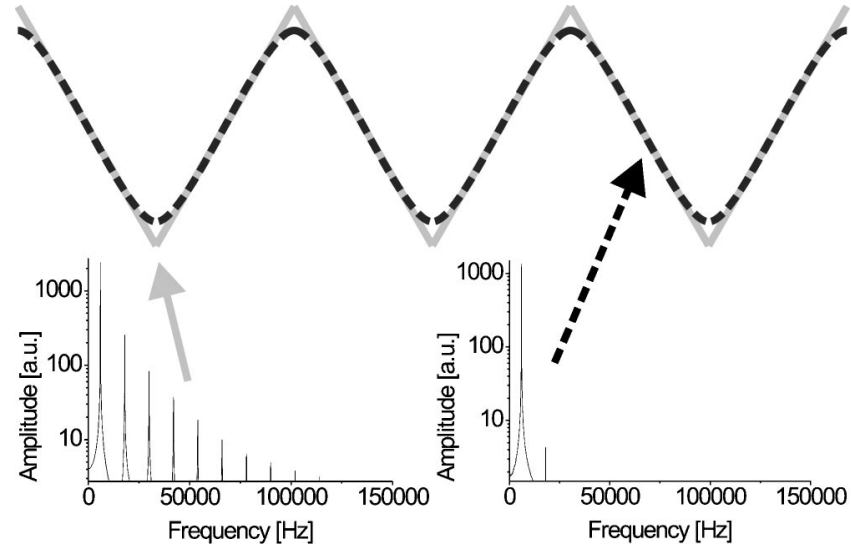

FIG. 9. Analog scan signal: without rounding (gray line) and with quadratic rounding of the tops (dashed line). The Fourier components of both signals are shown.

scan signal with a smoothly connected parabolic shape. This removes the spikes in acceleration at the sharp turning points and replaces them by well-defined regions of constant, low acceleration. The degree of rounding can be chosen by the software between $0 \%$ and $100 \%$ so that the user can adjust the rounding and optimize it for the line frequency at which he prefers to scan. The maximum rounding affects only $30 \%$ of the actual scan width so that the middle of the image is always distortion free and the pixel distances are equal, both in time and in position, which allows an easy analysis of distances, shapes, and time-dependent phenomena, such as surface diffusion. In addition to the rounding option, we have also developed a sine generator, which is used to completely remove all higher harmonics, for operation at the highest scan speeds.

The dashed line in Fig. 9 shows the analog triangular wave form with $100 \%$ rounding at the tops. The Fourier analysis of the wave form reveals that we introduce substantial amplitude only in the first (fundamental) and second harmonics to the scanner in this way.

We have connected the electronics to a variety of $\mathrm{SPMs}^{37}$ and find in practice that with the rounding of the triangular wave form the maximum scan rate at which these instruments can be operated without severe image distortions is increased by a factor of 5 .

\section{B. A new scanner design}

In this section we describe a new scan head with much higher resonance frequencies, which we have designed especially for testing the high-speed electronics. Currently, we are redesigning the scan head of our UHV variabletemperature STM for higher frequencies. ${ }^{38}$ The scan head contains a piezo stack that consists of two shear-mode piezos for the lateral motion $(X, Y)$, and one plate for the height $(Z)$ motion [see Figs. 10(a) and 10(b)]. The stack has a lateral dimensions of $5 \mathrm{~mm} \times 5 \mathrm{~mm}$, and a height of $3.5 \mathrm{~mm}$. The sample, in the present case HOPG, is glued on top of the piezo stack. The stack itself is glued onto a solid, stainless steel block, which can be moved with respect to the STM tip, which is in a tip holder in a second stainless steel block. The two blocks are attached to each other via a mechanically stiff
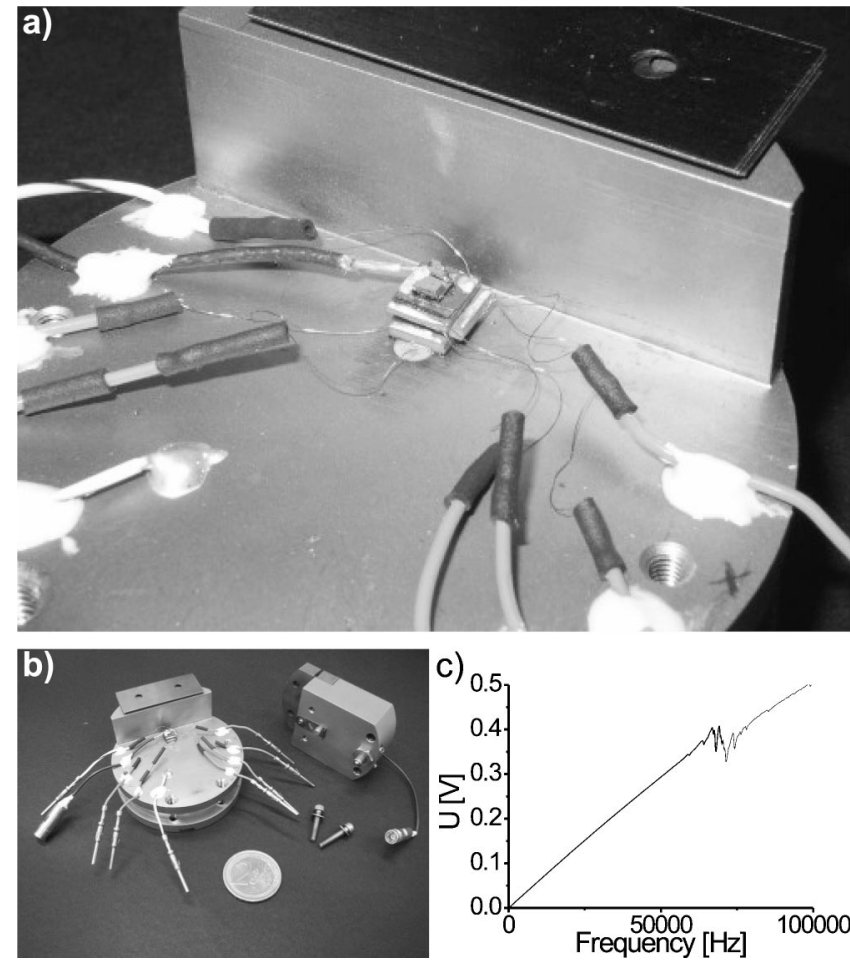

FIG. 10. (a), (b) Stack scanner with high resonance frequencies, a 2-Euro coin (25 mm diameter) is shown to illustrate the size; and (c) measurement of the mechanical resonances of this scanner.

leaf spring, which serves as a hinge and is used to allow the coarse, mechanical, tip-sample approach. Finite-element analysis of this scanner prdicts the lowest bending resonance frequency to be at approximately $\simeq 64 \mathrm{kHz}$. Due to a modest symmetry breaking we actually measure several different bending modes between $\sim 64$ and $\sim 75 \mathrm{kHz}$. The $Z$-resonance frequency lies above $100 \mathrm{kHz}$.

\section{High-speed imaging}

The new scanner, with its high resonance frequencies, allowed us to test our SPM control system up to its maximum speed. Figures 11(a) and 11(b) show atomically resolved STM images on HOPG. These images have been obtained at a frame rate of $80 \mathrm{~Hz}(12 \mathrm{~ms} /$ image $)$ for frames of $128 \times 128$ pixels. It is even possible to reach a frame rate of $188 \mathrm{~Hz}(5.3 \mathrm{~ms} /$ image $)$. But at such high frame rates we had to reduce the number of pixels per image to $256 \times 32$ [Fig.

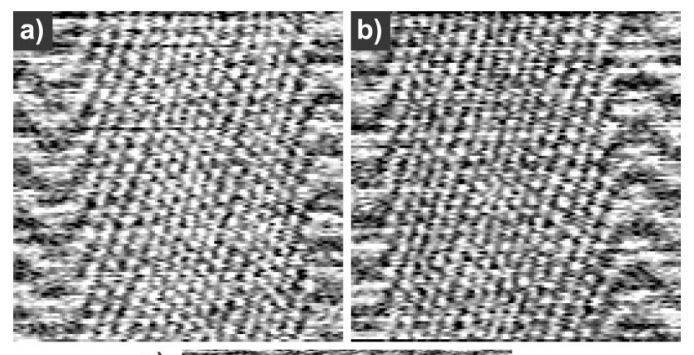

c)

FIG. 11. STM images $\left(U_{s}=-0.1 \mathrm{~V}\right.$ and $\left.\mathrm{I} \simeq 1 \mathrm{nA}\right)$ with atomic resolution on HOPG obtained at a frame rate of $80 \mathrm{~Hz}$ with $128 \times 128$ pixels: (a) trace (left to right), (b) retrace (right to left), and (c) at $188 \mathrm{~Hz}$ with 256 $\times 32$ pixels. 
11(c)], in order to stay within the bandwidth of the data bus of the control system. Although the stack scanner allows high scan speeds, its practical use is very limited for several reasons: (i) the scan sizes are very small, namely $300 \mathrm{~nm}$ in the $X / Y$ directions and only $200 \mathrm{~nm}$ in the $Z$ direction; (ii) the sample needs to be light and it has to be glued on the piezo stack; and (iii) the mechanical coarse approach is relatively crude.

\section{Lag of the piezo movement}

Figures 11(a) and 11(b) are trace and retrace images. The rounding of the triangular wave form can be seen at the edges of both images. Note that the distorted regions on the left side of image (a) and the right side of image (b) are wider than those at the opposite sides of the same images. This asymmetry is caused by a lag of the piezo movement with respect to the electronic drive signals. There are two reasons for this lag: mechanical hysteresis of the piezo element and a nonzero phase shift of the piezo response. If we would drive the piezo element at its mechanical bending resonance frequency, the response of the piezo element would lag behind with a phase shift of $90^{\circ}$. Of course, there is also a nonzero phase shift if we drive the piezo element below its eigenfrequency. The more we approach the eigenmode, the larger the lag. We observe similar effects in images where we excite the Z-resonance frequency of our scanner: there is a time delay between the maximum in the height $(Z)$ image and the corresponding error signal $(d Z)$.

The latter problem has been recognized before by Schitter et al., ${ }^{21}$ who have presented a first solution in the form of a compensation loop $\left(H_{\infty}\right.$ control).

\section{OUTLOOK}

The high-frequency scanner, used here for testing purposes, has several disadvantages such as its very small scan range. Currently, we are developing a high-frequency version of our UHV STM. ${ }^{38}$ Clearly, the mechanical behavior of SPMs can be improved in a variety of ways, for example by the miniaturization of the scan device or by the introduction of clever, mechanical compensation structures. With our UHV-STM, we intend to reach a frame rate of several tens of images per second routinely, without severe image distortion on a daily basis.

\section{ACKNOWLEDGMENTS}

This work is part of the research program of the Foundation for Fundamental Research on Matter (FOM) and was made possible by financial support from the Netherlands Organization for the Advancement of Research (NWO). T.H.O. acknowledges support from NWO, HFSP, and EU-FP6 (ASPRINT).

\footnotetext{
${ }^{1}$ R. Young, J. Ward, and F. Scire, Rev. Sci. Instrum. 43, 999 (1972).

${ }^{2}$ G. Binnig, H. Rohrer, Ch. Gerber, and E. Weibel, Phys. Rev. Lett. 49, 57 (1982).

${ }^{3}$ G. Binnig and H. Rohrer, IBM J. Res. Dev. 30, 4 (1986).
}

${ }^{4}$ K. Sagisaka1, M. Kitahara, D. Fujita, G. Kido, and N. Koguchi, Nanotechnology 15, S371 (2004).

${ }^{5}$ J. Wiebe, A. Wachowiak, F. Meier, D. Haude, T. Foster, M. Morgenstern, and R. Wiesendanger, Rev. Sci. Instrum. 75, 205411 (2004).

${ }^{6}$ M. J. Rost, D. A. Quist, and J. W. M. Frenken, Phys. Rev. Lett. 91, 026101 (2003)

${ }^{7}$ P. B. Rasmussen, B. L. M. Hendriksen, H. Zeijlemaker, H. G. Ficke, and J. W. M. Frenken, Rev. Sci. Instrum. 69, 3879 (1998).

${ }^{8}$ E. Laegsgaard, L. Osterlund, P. Thostrup, P. B. Rasmussen, I. Stensgaard, and F. Besenbacher, Rev. Sci. Instrum. 72, 3537 (2001).

${ }^{9}$ The Millipede: A future AFM-based data storage system from IBM; A. Pantazi, M. A. Lantz, G. Cherubini, H. Pozidis, and E. Eleftheriou, Nanotechnology 15, S612 (2004).

${ }^{10}$ MAPPER Lithography B.V. 〈http://www.mapperlithography.com/>

${ }^{11}$ J. Wilson and C. de Groot, J. Phys. Chem. 99, 7860 (1995).

${ }^{12}$ Dimension 5000, Digital Instruments, Veeco.

${ }^{13}$ L. Kuipers, M. S. Hoogeman, J. W. M. Frenken, and H. van Beijeren, Phys. Rev. B 52, 11387 (1995).

${ }^{14}$ M. J. Rost and J. W. M. Frenken, Phys. Rev. Lett. 87, 039603 (2001).

${ }^{15}$ L. Kuipers, R. W. M. Loos, H. Neerings, J. ter Horst, G. J. Ruwiel, A. P. de Jongh, and J. W. M. Frenken, Rev. Sci. Instrum. 66, 4557 (1995).

${ }^{16}$ T. Ando, N. Kodera, D. Maruyama, E. Takai, K. Saito, and A. Toda, Jpn. J. Appl. Phys., Part 1 41, 4851 (2002); PNAS 98, 12468 (2001).

${ }^{17}$ J. H. Kindt, G. E. Fantner, J. A. Cutroni, and P. K. Hansma, Ultramicroscopy 100, 259 (2004).

${ }^{18}$ R. Curtis, T. Mitsui, and E. Ganz, Rev. Sci. Instrum. 68, 2790 (1997).

${ }^{19}$ A. D. L. Humphris, J. K. Hobbs, and M. J. Miles, Appl. Phys. Lett. 83, 6 (2003).

${ }^{20}$ T. Michely, M. Kaiser, and M. J. Rost, Rev. Sci. Instrum. 71, 4461 (2000).

${ }^{21}$ G. Schitter, P. Menold, H. F. Knapp, F. Allgo, and A. Stemmer, Rev. Sci. Instrum. 72, 3320 (2001).

${ }^{22}$ STM feedback electronics typically contain a preamplifier, an absolute value converter, a logarithmic converter, proportional and integral gains, filters, and a high-voltage driver.

${ }^{23}$ L. Libioulle, A. Radenovic, E. Bystrenova, and G. Dietler, Rev. Sci. Instrum. 74, 1016 (2003).

${ }^{24}$ M. S. Hoogeman et al., Rev. Sci. Instrum. 69, 2072 (1998).

${ }^{25}$ Schroff GmbH, Germany 〈http://www.schroff.de〉.

${ }^{26}$ Our preamplifier is mounted directly next to the vacuum chamber to keep its capacitive input load as low as possible.

${ }^{27}$ See, e.g., Femto GmbH, RHK, ....

${ }^{28}$ We use first order filters with bandwidths of $0.3,1,3,10,30,100,300$, and $600 \mathrm{kHz}$.

${ }^{29}$ S. Hosaka, T. Hasegawa, S. Hosoki, and K. Takata, Rev. Sci. Instrum. 61, 1342 (1990)

${ }^{30}$ Strictly speaking, this calculation can be performed only if the work function is constant everywhere on the surface.

${ }^{31}$ The reason for this lies in the frequency dependence of the integral gain of the feedback electronics for frequencies below the mechanical resonance frequency of the piezo element and in the mechanical behavior of the piezo above its resonance.

${ }^{32}$ Supply voltages are controlled by DACs.

${ }^{33}$ In our case we have two piezo motors: one is used for the coarse approach, while the second is used for a lateral scanner positioning.

${ }^{34}$ See EPAPS Document No. E-RSINAK-76-283505 for 4 video files. This document can be reached via a direct link in the online article's HTML reference section or via the EPAPS homepage (http://www.aip.org/ pubservs/epaps.html).

${ }^{35}$ T. Michely, M. Kaiser, and M. J. Rost, Rev. Sci. Instrum. 71, 4461 (2000).

${ }^{36} \mathrm{We}$ used the commercially available software package FEMLAB from COMSOL, Inc.

${ }^{37}$ Until now we have connected five different SPMs: two commercial AFMs (the Nanoscope III from Digital Instruments and the Pico Scan from Molecular Imaging), one home-built AFM, and two home-built STMs.

${ }^{38}$ Currently we are working on a different design. We expect that this design will have a lowest resonance frequency of $\sim 100 \mathrm{kHz}$, an $X / Y$ range of $1 \mu \mathrm{m}$, and a $Z$ range of $400 \mathrm{~nm}$. 